\title{
Purposeful surgical delay and the coronavirus pandemic: how will black breast cancer patients fare?
}

\author{
Samilia Obeng-Gyasi ${ }^{1,2,3,7} \oplus$ Bridget Oppong ${ }^{1,2,3,7} \cdot$ Electra D. Paskett ${ }^{2,3,4,5} \cdot$ Maryam Lustberg $^{2,5,6,7}$
}

Received: 5 May 2020 / Accepted: 9 June 2020 / Published online: 16 June 2020

(c) Springer Science+Business Media, LLC, part of Springer Nature 2020

\begin{abstract}
Purpose The Coronavirus pandemic has exposed substantial racial and ethnic health and healthcare disparities. Black breast cancer patients face significant disparities in stage of presentation, surgical management, and mortality. The objective of this editorial is to examine the possible implications of the surgical delay imposed by the pandemic on black breast cancer patients.

Methods The American College of Surgeons, the Society of Surgical Oncology, and the American Society of Breast Surgeons recommendations for surgical delay during the Coronavirus Disease 2019 (COVID-19) were evaluated and discussed. Results Guidelines by major surgical organizations on surgical delay for breast cancer patients may inadvertently exacerbate disparities in time to surgery for black breast cancer patients. Our recommendations to better characterize the impact of these guidelines on surgical delay among vulnerable populations include the following: (1) track time from biopsy-proven diagnosis to surgery by race and ethnicity, (2) document patient and institution-related reasons for surgical delay, (3) record patient and disease-related variables/reasons for the selection of breast conservation surgery, mastectomy, and reconstruction by race and ethnicity, and (4) collect data on impactful social determinants of health such as financial reserve, housing conditions, stress, and transportation.

Conclusions The COVID-19 pandemic may exacerbate delays in time to surgery among black breast cancer patients. Surgeons should incorporate collection of social determinants of health into their clinical practice to better understand the impact of COVID-19 on racial and ethnic disparities in surgical management.
\end{abstract}

Keywords Breast cancer $\cdot$ Coronavirus pandemic $\cdot$ Racial disparities $\cdot$ Surgery

Samilia Obeng-Gyasi

samilia.obeng-gyasi@osumc.edu

1 Division of Surgical Oncology, Department of Surgery, The Ohio State University, Columbus, OH, USA

2 Comprehensive Cancer Center, The Ohio State University, Columbus, $\mathrm{OH}$, USA

3 Arthur G. James Cancer Hospital and Richard J. Solove Research Institute, Columbus, OH, USA

4 Division of Epidemiology, College of Public Health, The Ohio State University, Columbus, OH, USA

5 Division of Cancer Prevention and Control, Department of Internal Medicine, College of Medicine, The Ohio State University, Columbus, $\mathrm{OH}$, USA

6 Division of Medical Oncology, Department of Internal Medicine, College of Medicine, The Ohio State University, Columbus, OH, USA

7 Stefanie Spielman Comprehensive Breast Center, The Ohio State University Wexner Medical Center, Columbus, OH, USA

\section{Introduction}

As the USA comes to terms with the scope and magnitude of the current Coronavirus Disease 2019 (COVID-19) pandemic, an insidious long simmering problem has risen to the surface of the public consciousness. Preliminary data indicate black patients diagnosed with the virus have a more aggressive clinical course and a worse mortality than their white counterparts. ${ }^{1}$ For example, in some of the hardest hit areas such as New York, Michigan, and Illinois, blacks account for up to $30 \%$ of deaths. Furthermore, in Louisiana, $70 \%$ of coronavirus virus deaths are among blacks despite making up only $32 \%$ of the state's population [1]. Possible explanations for these disparities include higher underlying comorbidities, lack of or delayed access to healthcare, and receipt of lower quality care by black patients. Although these differences in clinical outcomes are alarming, for many in the field of health disparities research, these outcomes are 
not unexpected. For decades health disparities researchers have meticulously documented how structural racism has affected access to care and healthcare delivery resulting in racial and ethnic differences in presentation, management, and mortality for many acute and chronic illnesses. In oncology, racial, and ethnic health and healthcare disparities continue to influence clinical outcomes in both screen detectable (e.g., breast, prostate, colorectal, lung) and nonscreen detectable cancers (e.g., pancreatic cancer). Despite improvements in screening technology and increased participation in screening, black breast cancer patients continue to present with more advanced cancers and have higher mortality rates than their white counterparts [2]. This brief commentary examines the possible implications of purposeful surgical delay for black breast cancer patients within the context of the coronavirus pandemic.

\section{Racial disparities and clinical outcomes in breast cancer}

Black breast cancer patients are more likely to present with later stages of disease, have more aggressive breast cancer subtypes, such as triple negative breast cancer, and are diagnosed at younger ages than white women [2,3]. In addition, they have higher mortality rates than their white counterparts [2]. These disparities in clinical outcomes are a complex interplay between tumor biology, social determinants of health (SDH), and genetics [4]. Emerging fields such as human social genomics indicate these racial differences may be the result of interactions between genetic predisposition and genetic expression facilitated by environmental triggers such a racism, segregation, and poverty [5]

To date, multiple retrospective reviews of time to treatment have consistently shown black breast cancer patients are most likely to face treatment delays [6-8]. These treatment delays are significant as studies have shown patients with delays in time to surgery have a higher disease specific and overall mortality [6]. Furthermore, for breast cancer patients, the time intervals from first symptom presentation to biopsy and biopsy-proven diagnosis to treatment initiation are the most stressful times [9]. Explanations of surgical delay among black breast cancer patients include SDH such as insurance, education, access, transportation, and finances. Additionally, health system-based factors such as suboptimal doctor patient relationships, differing facilities for biopsy and definitive surgical treatment, and poorly managed underlying comorbidities requiring optimization prior to surgery also most likely contribute to surgical delay.

\section{Purposeful surgical delay and surgical disparities}

As statistics for the pandemic continue to evolve, emerging data indicate black patients are disproportionately hospitalized and have higher mortality rates than white patients [1]. In response to the pandemic, healthcare stakeholders and policymakers have instituted a series of public health initiatives and treatment recommendations to help reduce the spread of the virus and preserve valuable hospital resources. In March of 2020, the Centers for Medicare and Medicaid Services (CMS) announced that all elective surgeries and non-essential surgical procedures should be delayed during the coronavirus pandemic [10]. In response to this CMS recommendation, the American College of Surgeons (ACS), the Society of Surgical Oncology (SSO), and the American Association of Breast Surgeons (ASBrS) released guidelines for surgical delay among breast cancer patients. Unfortunately, these recommendations on surgical delay, though necessary, may inadvertently harm longterm oncological outcomes among patient populations that already have difficulty accessing healthcare and receiving high-quality care.

With significant delays in time to surgery at baseline, the purposeful delays imposed by the coronavirus may exacerbate underlying longer surgical wait times and subsequent worse clinical outcomes among black breast cancer patients. For example, current guidelines by various surgical organizations recommend consideration of surgical delay for clinical stage-one hormone receptor-positive breast cancer patients $[11,12]$. This recommendation, though appropriate, may adversely impact black breast cancer patients as studies have shown that even with favorable subtypes such as hormone receptor-positive cancer, black women continue to have a higher mortality rates than white women [13].

Additional recommendations include consideration of neoadjuvant endocrine therapy in clinically stage I-II patients with hormone receptor-positive tumors. Inherent in this recommendation is the assumption that treatment adherence is consistent across all racial and ethnic groups. However, in their systematic review of the impact of race on endocrine therapy adherence, Roberts et al. noted that black women have low rates of initiation and higher rates of nonadherence to endocrine therapy compared to other racial and ethnics groups [14].

Black women also have higher rates of triple negative breast cancer which frequently requires multimodal therapy $[2,15]$. The coordination of care when receiving treatment from multiple specialties is important to ensure timeliness of care especially in very tenuous circumstances such as this pandemic. Current guidelines recommend neoadjuvant chemotherapy for T2N0-3M0 or T0-4N1-3M0 patients with triple negative or HER $2+$ cancer which is consistent with the pre-Coronavirus pandemic practices [11]. However, we anticipate that the pandemic will unintentionally further exacerbate SDH such as access, transportation, income, stress, social networks, and finances which will contribute to diminished neoadjuvant chemotherapy adherence or 
result in an extended time period for administration. This may result in surgical delay, post-neoadjuvant chemotherapy, as patients attempt to navigate treatment side effects within the context of diminishing material resources (limited income, transportation issues, etc.) and public health initiatives such as social distancing. Moreover, these SDH will not only influence timeliness of operations but also will affect post-surgical care and follow-up clinic visits further worsening existing disparities in quality of care and subsequently mortality.

\section{Impact of reemergence from surgical delay}

As the pandemic curve flattens, states have started reemergence plans which also include guidelines for starting elective surgeries. To address this issue, multiple surgical societies in conjunction with the National Comprehensive Cancer Network and the American College of Radiology created a COVID-19 Pandemic Breast Cancer Consortium to provide guidance on the initiation of surgical management among breast cancer patients with surgical delay due to COVID19. Recommendations from the consortium include stratification of patients into high, intermediate, and low priority for treatment based on their breast disease, comorbidities, COVID-19 risk, and expected hospital resource utilization [16]. It is anticipated that this strategy, with clinician and patient buy in, will minimize the impact of COVID-19 on clinical outcomes by providing clear guidance on surgical prioritization. Additionally, the Massachusetts General Hospital (MGH) tool, created to help with surgical case prioritization, awards higher priority points to variables that portend a worse mortality such as younger age, larger tumor size, increasing time since biopsy, high genomic risk, and hormone receptor-negative disease which are all disproportionally found in black women [17, 18]. Of note, the MGH tool determines the risk of delaying breast cancer surgery based on multiple domains including but not limited to: neoadjuvant chemotherapy, neoadjuvant endocrine therapy, margin status, histology, genomic risk, hormone receptor status, tumor size, age, physical exam, and imaging response to neoadjuvant chemotherapy or endocrine therapy, hormone receptor status, and time since biopsy [16].

In order to optimize this reemergence strategy for black breast cancer patients, providers and hospitals will need to be acutely aware of institutional barriers (e.g., surgeon and operating room availability, radiology services for localization device placement and personal protective equipment and so on) and patient-related barriers (e.g., fear, transportation, economic instability, poor social networks, or support) that might prevent timely surgical management. We anticipate that utilization of the prioritization strategy provided by the consortium in conjunction with the MGH tool may actually mitigate some of the disparities in time to surgery faced by black breast cancer patients pre-COVID-19. Specifically, prioritization of patients with more aggressive tumor biology will inadvertently force healthcare providers and hospitals to focus more acutely on time to surgery among black breast cancer patients due to their higher incidence of aggressive breast disease. Essentially, this prioritization strategy may be leveraged to reduce racial and ethnic disparities in time to surgery.

To address patient-related barriers to delay such as transportation, economic instability, lodging for patients traveling from far distances and stress incorporation of patient, and/ or financial navigators should be considered. Patient navigation has been shown to improve treatment adherence and clinic appointment attendance, increase completion of cancer directed therapy, and improve patient satisfaction and is also cost-effective $[19,20]$. The literature on financial navigation is still emerging as it is a relatively new navigation paradigm. Financial navigators help patients navigate institutional, community, and state resources for treatmentrelated out-of-pocket costs and also screen for financial distress [21]. For institutions with resources such as financial counselors and social workers, clinic workflows should be optimized for easy patient access and follow-up to these resources. We anticipate that integration of these resources into clinic workflows will also improve collection of data on SDH.

\section{Future directions}

Although the current breast surgical oncology guidelines are necessary to mitigate the effects of the coronavirus pandemic, they may expose deeply rooted racial and ethnic disparities in timeliness of surgical care and clinical outcomes among breast cancer patients. For black breast cancer patients who bear the brunt of these disparities, purposeful surgical delays may widen already established disparities in presentation, surgical management, and mortality. To better understand the effects of the coronavirus pandemic on surgical outcomes in breast cancer patients, we recommend better SDH data collection during this pandemic ("Box 1" section) and offer ways to improve SDH data collection ("Box 2" section).

Current predictions indicate infections from the coronavirus may continue for some time. Reemergence strategies focused on patients with more aggressive disease coupled with plans to mitigate patient-related factors for surgical delay (e.g., transportation) will be the most impactful approach to reducing surgical delays in black breast cancer patients. The widely persistent and pervasive racial and ethnic disparities being exposed by the virus provide surgeons an opportunity to collect and reconceptualize SDH and their implications for surgical disparities. 


\section{Box 1: recommendations to characterize racial and ethnic surgical disparities during coronavirus pandemic}

1. Track time from biopsy-proven diagnosis to surgery by race and ethnicity.

2. Document patient and institution-related reasons for surgical delay.

3. Record patient and disease-related variables/reasons for the selection of breast conservation, mastectomy, and reconstruction by race and ethnicity.

4. Collect data on impactful social determinants of health such as financial reserve, housing conditions, stress, and transportation

\section{Box 2: recommendations to integrate SDH collection during COVID-19 into clinical practice}

1. Include questions on social determinants of health (SDH) into clinic check-in workflow.

2. Routinely ask questions on SDH as part of social history intake during history and physical exam. Repeat questions on subsequent clinic visits.

3. Integrate SDH questions into the electronic medical record.

4. If available, consider incorporating patient navigators, financial navigators, financial counselors, or social workers into all clinic visits.

\section{Funding None}

\section{Compliance with ethical standards}

Conflict of interest All authors declares that they have no conflicts of interest.

Ethical approval This article does not contain any studies with human participants or animals performed by any of the authors.

Informed consent This article does not contain any studies with human participants thus no informed consent was needed

\section{References}

1. Berry D (2020) Why are so many black people dying from coronavirus?. USA Today, USA Today

2. DeSantis CE, Ma J, Goding Sauer A et al (2017) Breast cancer statistics, 2017, racial disparity in mortality by state. CA Cancer J Clin 67:439-448

3. Hung MC, Ekwueme DU, Rim SH et al (2016) Racial/ethnicity disparities in invasive breast cancer among younger and older women: An analysis using multiple measures of population health. Cancer Epidemiol 45:112-118
4. Newman LA (2017) Breast Cancer Disparities: Socioeconomic Factors versus Biology. Ann Surg Oncol 24:2869-2875

5. Linnenbringer E, Gehlert S, Geronimus AT (2017) Black-White Disparities in Breast Cancer Subtype: The Intersection of Socially Patterned Stress and Genetic Expression. AIMS Public Health 4:526-556

6. Bleicher RJ, Ruth K, Sigurdson ER et al (2016) Time to Surgery and Breast Cancer Survival in the United States. JAMA Oncol 2:330-339

7. Fedewa SA, Edge SB, Stewart AK et al (2011) Race and ethnicity are associated with delays in breast cancer treatment (2003-2006). J Health Care Poor Underserved 22:128-141

8. George P, Chandwani S, Gabel M et al (2015) Diagnosis and surgical delays in African American and white women with earlystage breast cancer. J Womens Health (Larchmt) 24:209-217

9. Ganz PA (2000) Quality of Life Across the Continuum of Breast Cancer Care. Breast J 6:324-330

10. Services CfMaM: CMS Releases Recommendations on Adult Elective Surgeries, Non-Essential Medical, Surgical, and Dental Procedures During COVID-19 Response, Centers for Medicare and Medicaid Services, 2020

11. Oncology. SoS: Resource for Management Options of Breast Cancer During COVID-19, 2020

12. Dietz JY, K., Krutzman, s., Anderson B., Willey, S., Boolbol, S., Bleicher, R. Sarantou T., Baron, P. Isakoff, S. Gradishar, W. Burstein, H. Schulman, L., Moran, M., Stevens, R., Lyons, J.: Recommendations for Prioritization, Treatment and Triage of Breast Cancer Patients During the COVID-19 Pandemic: Executive Summary, 2020

13. O'Brien KM, Cole SR, Tse C-K et al (2010) Intrinsic Breast Tumor Subtypes, Race, and Long-Term Survival in the Carolina Breast Cancer Study. Clin Cancer Res 16:6100-6110

14. Roberts MC, Wheeler SB, Reeder-Hayes K (2015) Racial/Ethnic and socioeconomic disparities in endocrine therapy adherence in breast cancer: a systematic review. Am J Public Health 105(Suppl 3):e4-e15

15. Newman LA, Kaljee LM (2017) Health Disparities and TripleNegative Breast Cancer in African American Women: A Review. JAMA Surg 152:485-493

16. Monticciolo L. Debra PMD, Shulman N. Lawrence, Baron L. Paul, Bleicher J. Richard, Kurtzman H., Scott, Sarantou Terry, Stevens E. Randy, Yao A. Katherine, Boolbol K. Susan, Dietz R., Jill, Smith L. Barbara, Specht C. Michelle, Wiley C. Shawna, Martin Miguel, Anderson: COVID-19 Pandemic Breast Cancer Consortium's Considerations for Re-entry, 2020

17. DeSantis CE, Miller KD, Goding Sauer A, et al: Cancer statistics for African Americans, 2019. CA: A Cancer Journal for Clinicians 69:211-233, 2019

18. Daly B, Olopade OI (2015) A perfect storm: How tumor biology, genomics, and health care delivery patterns collide to create a racial survival disparity in breast cancer and proposed interventions for change. CA Cancer J Clin 65:221-238

19. Bernardo BM, Zhang X, Beverly Hery CM et al (2019) The efficacy and cost-effectiveness of patient navigation programs across the cancer continuum: A systematic review. Cancer 125:2747-2761

20. Krok-Schoen JL, Oliveri JM, Paskett ED: Cancer Care Delivery and Women's Health: The Role of Patient Navigation. Frontiers in Oncology 6, 2016

21. Company. TAB: Cancer Patient Financial Navigation: Helping Patients Manage Their Costs While Protecting Program Margins., in Deirdre. S (ed): Cancer Patient Financial Navigation, 2014

Publisher's Note Springer Nature remains neutral with regard to jurisdictional claims in published maps and institutional affiliations. 